\title{
Summary of: Estimation of remaining dentine thickness below deep lesions of caries
}

\author{
P. E. Lancaster, ${ }^{1}$ H. L. Craddock ${ }^{2}$ and F. A. Carmichael ${ }^{3}$
}

\section{FULL PAPER DETAILS}

${ }^{1 * D e p a r t m e n t ~ o f ~ R e s t o r a t i v e ~ D e n t i s t r y, ~}{ }^{2}$ Senior Lecturer and Honourary Consultant, Department of Fixed and Removable Prosthodontics, ${ }^{3}$ Department of Oral and Maxillofacial Radiology, Leeds Dental

Institute, University of Leeds, Worsley Building

Clarendon Way, Leeds, LS2 9LU

${ }^{*}$ Correspondence to: Miss Paula E. Lancaster

Email: p.e.lancaster@leeds.ac.uk

Online article number E20

Refereed Paper - accepted 4 March 2011

DOI: $10.1038 /$ sj.bdj.2011.968

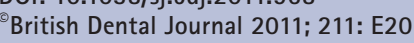

Objective The objective of this study was to investigate agreement between the estimated remaining dentine thickness (RDT) under lesions of caries, measured from a conventional in vivo periapical radiograph, compared directly to the measured RDT of the tooth. Additional investigation was to be made for agreement between in vitro digital radiographs and conventional radiographs. Design and setting This was a cross-sectional, single centre study at Leeds Dental Institute, United Kingdom, in 2009. Subjects, materials and methods Twenty-five carious teeth with occlusal or proximal lesions were collected from patients aged 19 to 82 years attending the Oral Surgery Department. Each patient had a pre-extraction in vivo periapical radiograph of the tooth demonstrating an intact layer of dentine below the lesions of caries. Postextraction in vitro digital and conventional radiographs were taken. Main outcome measures Agreement of the RDT was analysed using Bland-Altman plots. Results A trend for the radiographic images to over-estimate the RDT compared to the tooth was found. Greater over-estimation of the RDT by conventional radiographs both in vivo and in vitro was shown compared to the in vitro digital images in the majority of cases. Conclusion This analysis has demonstrated it is not possible to estimate the dimension of the RDT from a periapical radiograph.

\section{EDITOR'S SUMMARY}

One of the key procedures that we undertake in patient care is diagnosis. The more accurate this is, the greater are the chances of providing the appropriate treatment with consequent improved prognosis and outcome. The diagnosis of caries has ever been a complex and difficult task but with the advent of finer and finer methods of caries removal and improved restorative materials (minimal intervention) the need for more refined diagnosis has also emerged.

Traditionally, the use of radiographs has been a crucial diagnostic tool for identifying either new or recurrent caries but the extent to which this accurately pinpoints the remaining dentine thickness (RDT), as described in this paper, seems to be equivocal. This study is rare in that it combines the use of both in vivo information from radiographs taken before the extraction of a tooth and then further in vitro radiographic and histological information garnered after extraction. In normal clinical practice this would not be possible. The closest we could otherwise get to this would be opening and excavation of the affected tooth but the remaining caries thickness could only be an estimation based on eyesight and experience, with a halt to excavation dictated by the chosen method of restoration, lining, pulp capping or root canal therapy.

The results of this work indicate that caries is invariably deeper in reality than is shown on periapical radiographs and this is an important finding in guiding our clinical decision making. It may well be that with the continuing developments in imaging technology we will eventually have the ability to measure the extent of caries and RDT to smaller and smaller tolerances but the value of being able to do so will also have to be matched with techniques and materials to safeguard the pulp from injury to a similar finer extent. It may well be that we are reaching the extent of our ability to intervene biologically at this interface but the knowledge gained from this paper will enable us to make more informed choices in patient care.

The full paper can be accessed from the $B D J$ website (www.bdj.co.uk), under 'Research' in the table of contents for Volume 211 issue 10.

Stephen Hancocks Editor-in-Chief

DOI: 10.1038/sj.bdj.2011.985 
TO ACCESS THE BDJ WEBSITE TO READ THE FULL PAPER:

- BDA Members should go to www.bda.org.

- Click the 'login' button on the right-hand side and enter your BDA login details.

- Once you have logged in click the 'BDJ' tab to transfer to the BDJ website with full access.

IF YOUR LOGIN DETAILS DO NOT WORK:

- Get a password reminder: go to www.bda.org, click the login button on the right-hand side and then click the forgotten password link.

- Use a recommended browser: we recommend Microsoft Internet Explorer or Mozilla Firefox.

- Ensure that the security settings on your browser are set to recommended levels.

IF YOU HAVE NOT YET SIGNED UP TO USE THE BDA WEBSITE:

- Go to www.bda.org/getstarted for information on how to start using the BDA website.
IN BRIEF

- Stepwise excavation is an accepted technique for restoring deep caries lesions.

- The remaining dentine thickness influences pulp vitality and the outcome of certain restorative procedures.

- Reliable measurement of tooth dimensions from an intra-oral conventional or digital radiograph was not achievable.

- The general trend for the radiographic images was to over-estimate the remaining dentine thickness.

\section{COMMENTARY}

This paper adds to the discussion of how deep the carious process may proceed before irreversible pulpal inflammation occurs. A parameter in this dispute is the remaining dentine thickness (RDT) between the carious lesion and the pulp chamber.

As early as 1966, Reeves and Stanley ${ }^{1}$ stated that if bacterial penetration had progressed within $0.5 \mathrm{~mm}$ of the pulp, irreversible pulpitis occurs as reaction. In a more recent study, Murray et $a .^{2}$ investigated the relation between RDT and pulp inflammation. The pulpal reaction was moderate when RDT was around $0.6 \mathrm{~mm}$ and became worse when RDT decreased to $0.4 \mathrm{~mm}$.

If a clinician could accurately determine the RDT in the most advanced area of the caries lesion, this could provide him or her with information about the state of pulpal health and thereby could help in the clinical decision making process. In the present study the value of radiographic images in determining the RDT is investigated by comparing measurements taken from periapical radiographs and from histological sections of the tooth. Unfortunately, due to ethical reasons, no bite wing radiographs could be used.

The results show that it is not possible to accurately estimate the RDT from a periapical radiograph. Regardless of the image technique used, whether conventional or digital radiography, it leads to an overestimation of the RDT compared to the actual thickness assessed on the histological sections. For the clinician this is valuable information to take into account when examining radiographs of deep dentinal lesions.

It would be valuable to have information on the clinical history and reasons for extraction of the teeth used, and interesting to correlate the pain history with the assessed RDT on the histological sections of the teeth.

This study shows the limitations of the use of periapical radiographs in assessing the remaining dentine thickness in deep carious lesions. To elucidate this question other techniques like optical coherence tomography (OCT) or cone beam CT (CBCT) could be of value.

\section{A. J. P. van Strijp}

Chair, Department of Cariology, Endodontology and Pedodontology, Academic Centre for Dentistry Amsterdam (ACTA), The Netherlands

1. Reeves R, Stanley H R. The relationship of bacterial penetration and pulpal pathosis in carious teeth. Oral Surg Oral Med Oral Pathol 1966; 22: 59-65.

2. Murray P E, Smith A J, Windsor L J, Mjör I A. Remaining dentine thickness and human pulp responses. Int Endod J 2003; 36: 33-43.

\section{AUTHOR QUESTIONS AND ANSWERS}

1. Why did you undertake this research? Stepwise excavation aims to retain a layer of dentine, avoiding pulpal exposures. This has been shown to influence the long-term vitality of the tooth, but how do we know when to stop excavating, and is there a simple method of measuring the remaining dentine thickness? It is generally accepted that the size of carious lesions are underestimated on radiographs, which raised the issue: was the depth of the remaining dentine over-estimated on radiographs? No literature or articles were found to support this assumption.

A variety of methods have been explored to measure the depth of remaining dentine but none have proved reliably accurate. The most readily available image showing the remaining dentine depth was the radiograph and this is available to all practitioners. If the radiograph could be used to measure this dimension, the number of exposures may be reduced when excavating caries, thus improving the tooth's prognosis.

2. What would you like to do next in this area to follow on from this work?

There are imaging systems available for use in medicine and veterinary medicine which, to date, have not been used in dentistry, and these could hold the answer to reliably measure such dimensions as the remaining dentine depth. We hope to explore some of the alternative options and see if there are any advantages over the systems presently available for the dental practitioner. 